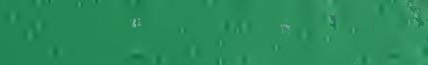

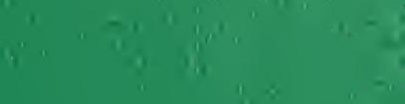

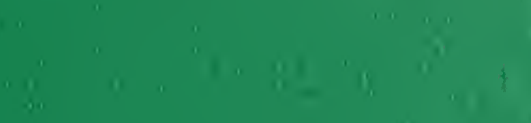
an

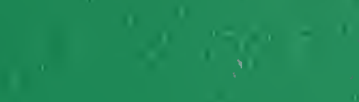

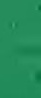

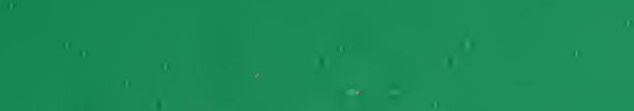


Digitized by the Internet Archive in 2012 with funding from Royal Ontario Museum 


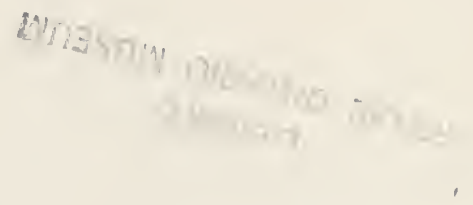

$$
\begin{aligned}
& \text { LIBRGIO MUSEUM } \\
& \text { ROYAL ONTARIO. MUL }
\end{aligned}
$$





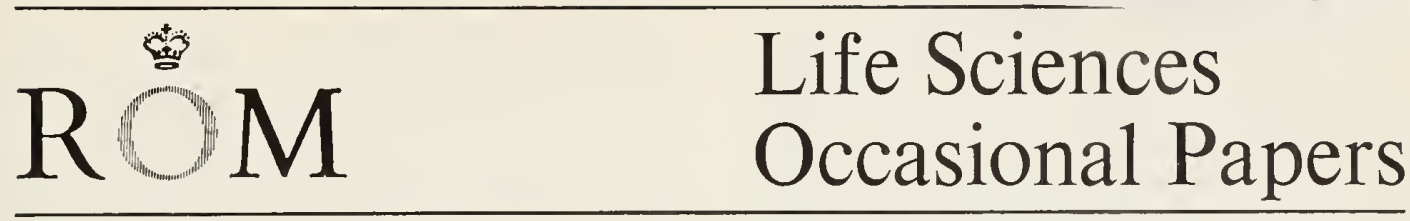

Royal Ontario Museum

June 19, 1974

No. 25

\section{A New Subspecies of the Bat Eumops auripendulus (Chiroptera: Molossidae), from Argentina and Eastern Brazil ${ }^{1}$}

by Judy L. Eger ${ }^{2}$

Abstract-The mastiff bat, Eurnops auripendulus (Chiroptera: Molossidae) occurs from southern Mexico to northeastern Argentina. In eastern Brazil and northeastern Argentina is a population that is morphologically distinct from other Eumops auripendulus. The new subspecies is geographically isolated from, and larger than Eumops a. auripendulus which occurs from the Amazon region of South America to southern Mexico.

[Chiroptera; Molossidae; Eumops auripendulus; subspecies novum; systematics.]

The mastiff bat, Eumops auripendulus (Shaw), first named the slouch-eared bat by Pennant (1793), was described by him from a drawing made by Buffon in 1789 (see Goodwin, 1960). Subsequently, Shaw (1800) renamed this species Vespertilio auripendulus. The specimen sketched by Buffon (1789) was from Cayenne, French Guiana, and therefore Cayenne is the type locality. According to Husson (1962), authors publishing since 1876 considered Dysopes $a b$ rasus Temminck (1827) to be the same species as $V$. auripendulus. But Husson (1962) extracted the skull of the type of $D$. abrasus and demonstrated that this speci- men was not a Eumlops but actually belonged to the genus Cynomops Thomas (1920). Thus Goodwin (1960) reassigned V. auripendulus to $E$. auripendulus. Consequently, $E$. auripendulus should be the name applied to these bats from the Guianas region.

Eumops auripendulus occurs from southern Mexico south to northern Argentina, and two specimens are known from Jamaica (9.1.4.48, British Museum (Natural History); no number, Institute of Jamaica). Sanborn (1932), in his review of the genus Eumops, listed two races of E. abrasus $(=E$. auripendulus) and assigned four specimens from eastern Brazil to E. abrasus abrasus and all other specimens to E. abrasus milleri. As $E$. auripendulus is the valid specific name, $E$. abrasus milleri is therefore synonymous with $E$. auripendulus auripendulus. In a recent review of the genus Eumops (Eger, 1973), I confirmed that Sanborn was correct in recognizing a distinct, isolated population of E. auripendulus in eastern Brazil. In this study 14 additional specimens of this population were identified and the distribution of the new subspecies, described herein, is known to extend southward to Misiones, Argentina.

${ }^{1}$ Based in part on a thesis submitted in partial fulfilment for the degree of M.Sc., Department of Zoology, University of Toronto, October 1973.

2 Department of Mammalogy, Royal Ontario Museum, Toronto. 
Materials and Methods-The 305 specimens examined were either study skins and skulls or specimens preserved in alcohol (some with skulls removed). Specimens examined are in the collections of the following institutions: American Museum of Natural History (AMNH); Academy of Natural Sciences of Philadelphia (ANSP); British Museum (Nat. Hist.) (BMNH); Carnegie Museum (CM); Field Museum of Natural History (FMNH); Institute of Jamaica ( $\mathrm{IJ}$ ); The Museum of Natural History, University of Kansas (KU); Los Angeles County Museum (LACM); Museum of Comparative Zoology, Harvard University (MCZ); Muséum National d'Histoire Naturelle, Paris and Brunoy (MNHN); Museum of Vertebrate Zoology, University of California, Berkeley (MVZ); Rijksmuseum van Naturlijke Historie, Leiden (RMNH); Royal Ontario Museum ( ROM); Texas Cooperative Wildlife Research Collection, Texas A\&M University (TCWC); Universidade de São Paulo (USP); Universidad Nacional Autónoma de México (UNAM); and the National Museum of Natural History (USNM).

Thirty-eight morphometric characters were studied. Measurements, in millimetres $(\mathrm{mm})$, were taken with dial calipers to the nearest $0.1 \mathrm{~mm}$ for wing bones, to the nearest $1.0 \mathrm{~mm}$ for body measurements, and to the nearest $0.05 \mathrm{~mm}$ for cranial measurements. The length of the tibia, often difficult to measure, was verified by radiographs where possible. Descriptions of pelage colour follow those given by Ridgway (1912).

Conventional morphological characters employed in chiropteran systematics were measured and are: External - Forearm (FOAR); metacarpals 3, 4, and 5 (3DME, 4DME, 5DME); phalanges 1 and 2 ( I PHL, 2PHL) of third, fourth and fifth digits; total length (TLEN); tail vertebrae (TVER); hind foot, including claws (HDFT); ear from notch (EARS); tragus (TRAG); and tibia (TIBI). Mandible - Condyloincisive length (CIMA); greatest length (GRLG), measured from one condyle to anterior face of incisors; canine to third molar ( $\left.\mathrm{C}-\mathrm{M}_{3}\right)$; width across canines $\left(\mathrm{C}_{1}-\mathrm{C}_{1}\right)$, including cingula; and height of lower canines (HTLC), mea- sured bucally from base of cingulum to crown. Broken or obviously worn teeth were not measured. Skull - Width of septum between basisphenoid pits (WBSP); length of basisphenoid pit (LBSP); total length (TOLG), measured from lambdoidal crest to anterior face of incisors; condyloincisive length (CBLG); palatal length (PALA), measured from anterior face of incisor to posterior margin of palate, lateral to posteromedial projection; zygomatic width (ZYGO); mastoid width (MAST); lachrymal width ( $\mathrm{LACH}$ ); interorbital width (IOWI); height of braincase (HBCA), measured from ventral border of foramen magnum to top of skull, not including sagittal crest; width across third molars $\left(\mathrm{M}^{3}-\mathrm{M}^{3}\right)$; canine to third molar $\left(\mathrm{C}-\mathrm{M}^{3}\right)$; width across canines $\left(\mathrm{C}^{1}-\mathrm{C}^{1}\right)$, including cingula; height of upper canines (HTUC), measured bucally from base of cingulum to crown; postorbital constriction (POCO); breadth of braincase (BBC1), anterior width of braincase measured where zygoma anastomose with parietals; and breadth of braincase (BBC2), measured dorsal to auditory bullae with blades of calipers resting on zygoma.

Localities of E. auripendulus (Fig. 1) are of specimens examined, records of immature specimens, and records from the literature. A study of sexual dimorphism of eight taxa indicated that male and female Eumops were dimorphic in a minimum of 26 per cent of the characters (Eger, 1973). Means and standard errors (S.E.) of all characters were calculated separately for males and females.

As E. a. auripendulus varies clinally, increasing in size from north to south (Eger, 1973), specimens from eastern Brazil and northern Argentina were compared only with those from the adjacent Amazon region of Brazil. Differences in character means were examined using Student's $t$-test (Sokal and Rohlf, 1969). Asterisks denote probability values as follows: $* * *=P \leqslant 0.001 ; * *=$ $P \leqslant 0.01 ; *=P \leqslant 0.05$; and $\mathrm{ns}=P>0.05$.

\section{Eumops auripendulus major ssp. nov.}

Holotype-ROM 50196, adult male, skin and skull, collected 17 April 1968 by H. Delpietro, purchased from Abel Fornes. 


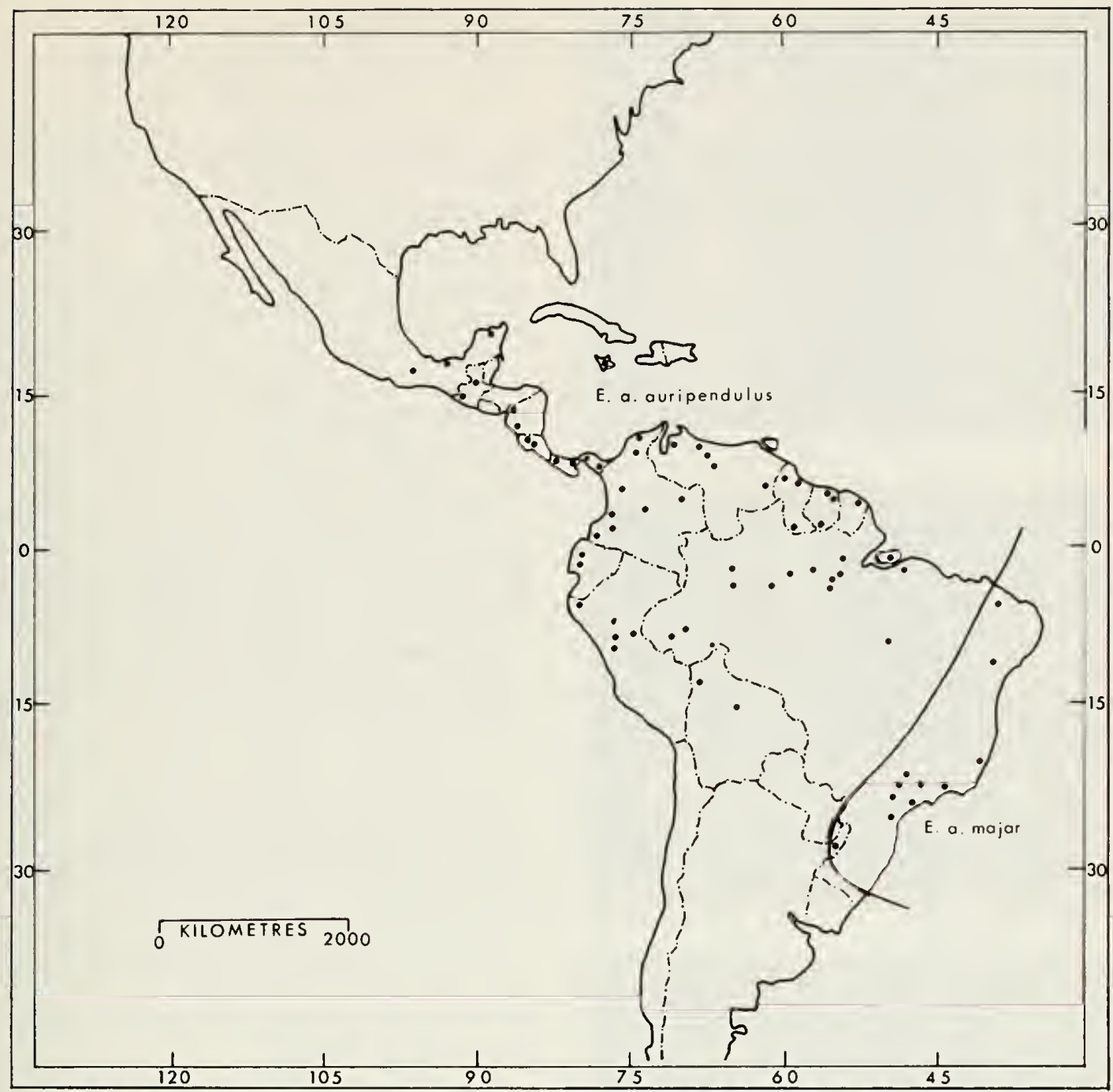

Fig. 1-Distribution of Eumops a. auripendulus and E. a. major in Middle and South America as determined from localities of specimens examined and from literature records.

Type locality-Campo Viera $\left(27^{\circ} 15^{\prime} \mathrm{S}\right.$, $\left.55^{\circ} 10^{\prime} \mathrm{W}\right)$, Misiones, Argentina.

Distribution-Northeastern Argentina and eastern Brazil (Fig. 1).

Description-Dorsal pelage Blackish-brown with pale basal band; ventral pelage Mummy Brown with white basal band; pinnae broad, arising from single point on forehead, united at base; tragus small and pointed; antitragus twice as broad as high; wing membranes furred proximal to forearm and on proximal ends of metacarpals; guard hairs on feet long. Dental formula $\frac{1}{2} \frac{1}{1} \frac{2}{2} \frac{3}{3}$; first upper premolar small, external to toothrow; canine and second premolar in contact; third commissure of third upper molar absent; basisphenoid pits shallow but well defined (Fig. 2B). Measurements of the holotype-See Table 1. Comparison-Eumops auripendulus major is similar in colour to E. a. auripendulus but statistically significantly larger in 27 of 38 characters in males and in 31 of 38 characters in females (Tables 1 and 2). Measurements of FOAR, 3DME, CIMA, GRLG, $\mathrm{C}-\mathrm{M}_{3}, \mathrm{C}-\mathrm{M}^{3}$ (females only), TOLG, and CBLG do not overlap in specimens of the two subspecies.

Specimens examined-(Those used in analysis marked with $\dagger$ ) 

and male E. a. major from eastern Brazil and northeastern Argentina

\begin{tabular}{|c|c|c|c|c|c|c|c|c|}
\hline \multirow[b]{2}{*}{ Character } & \multicolumn{3}{|c|}{ E. a. auripendulus } & \multirow[b]{2}{*}{$\begin{array}{c}\text { Holotype } \\
\text { ROM } 50196 \text { o }\end{array}$} & \multicolumn{3}{|c|}{ E. a. major } & \multirow[b]{2}{*}{$\begin{array}{l}\text { Student's } \\
t \text { value }\end{array}$} \\
\hline & $\mathrm{n}$ & Mean & \pm S.E. & & $\mathrm{n}$ & Mean & \pm S.E. & \\
\hline FOAR & 14 & 58.6 & 0.33 & 65.0 & 8 & 64.2 & 0.70 & $7.24^{* * *}$ \\
\hline 3DME & 16 & 60.6 & 0.46 & 65.7 & 8 & 66.1 & 0.49 & 7. $38^{* * *}$ \\
\hline $1 \mathrm{PHL}$ & 16 & 25.5 & 0.21 & 28.3 & 8 & 28.3 & 0.30 & $7.91^{* * *}$ \\
\hline 2PHL & 16 & 23.3 & 0.22 & 23.7 & 8 & 25.6 & 0.37 & $5.65^{* * *}$ \\
\hline 4DME & 16 & 58.4 & 0.50 & 62.3 & 8 & 63.4 & 0.42 & $7.66^{* * *}$ \\
\hline 1PHL & 16 & 21.5 & 0.21 & 23.0 & 8 & 23.9 & 0.39 & $6.10^{* * *}$ \\
\hline $2 \mathrm{PHL}$ & 16 & 5.7 & 0.17 & 6.0 & 8 & 6.1 & 0.20 & $1.38 \mathrm{~ns}$ \\
\hline 5DME & 16 & 32.8 & 0.25 & 35.3 & 8 & 35.9 & 0.54 & $5.30^{* * *}$ \\
\hline 1PHL & 16 & 17.6 & 0.15 & 19.4 & 7 & 19.3 & 0.24 & $6.15^{* * *}$ \\
\hline $2 \mathrm{PHL}$ & 16 & 5.5 & 0.07 & 5.0 & 8 & 6.0 & 0.20 & $2.42 *$ \\
\hline TLEN & 2 & 146.0 & - & 149.0 & 5 & 144.0 & 2.86 & - \\
\hline TVER & 4 & 47.8 & 2.84 & 55.0 & 6 & 55.2 & 2.02 & $2.19 \mathrm{~ns}$ \\
\hline HDFT & 6 & 11.7 & 0.76 & 11.0 & 5 & 12.6 & 1.03 & $0.74 \mathrm{~ns}$ \\
\hline EARS & 1 & 21.0 & - & 19.0 & 4 & 19.5 & 1.26 & $0.52 \mathrm{~ns}$ \\
\hline TRAG & 0 & - & - & 3.0 & 3 & 3.7 & 0.67 & - \\
\hline TIBI & 10 & 16.1 & 0.25 & 18.4 & 6 & 18.3 & 0.44 & $4.79^{* * *}$ \\
\hline HTLC & 6 & 4.1 & 0.03 & 4.8 & 4 & 4.8 & 0.08 & $7.35^{*}$ \\
\hline HTUC & 5 & 4.2 & 0.08 & 4.6 & 4 & 4.6 & 0.12 & $3.31 *$ \\
\hline WBSP & 6 & 0.6 & 0.04 & 0.7 & 6 & 0.8 & 0.06 & $2.57^{*}$ \\
\hline LBSP & 6 & 1.8 & 0.12 & 2.2 & 6 & 2.0 & 0.15 & $1.21 \mathrm{~ns}$ \\
\hline CIMA & 5 & 17.4 & 0.16 & 18.9 & 5 & 18.7 & 0.14 & $6.03^{* * *}$ \\
\hline GRLG & 6 & 17.8 & 0.17 & 19.4 & 6 & 19.1 & 0.15 & $5.48^{* * *}$ \\
\hline $\mathrm{C}-\mathrm{M}_{3}$ & 6 & 10.6 & 0.09 & 11.7 & 6 & 11.4 & 0.09 & $6.26^{* * *}$ \\
\hline $\mathrm{C}_{1}-\mathrm{C}_{1}$ & 6 & 3.9 & 0.19 & 4.5 & 9 & 3.9 & 0.06 & $2.11 \mathrm{~ns}$ \\
\hline TOLG & 6 & 25.4 & 0.11 & 27.1 & 6 & 27.2 & 0.23 & $7.30^{* * *}$ \\
\hline CBLG & 5 & 23.4 & 0.24 & 25.1 & 6 & 25.0 & 0.15 & $5.94^{* * *}$ \\
\hline PALA & 6 & 11.3 & 0.08 & 12.1 & 6 & 12.1 & 0.04 & $8.72^{* * *}$ \\
\hline ZYGO & 6 & 14.4 & 0.15 & 15.7 & 6 & 15.4 & 0.10 & $5.21^{* * *}$ \\
\hline MAST & 5 & 12.6 & 0.12 & 12.8 & 5 & 13.1 & 0.15 & $2.82^{*}$ \\
\hline LACH & 6 & 8.2 & 0.21 & 8.3 & 6 & 8.6 & 0.08 & $1.50 \mathrm{~ns}$ \\
\hline IOWI & 6 & 7.9 & 0.14 & 8.2 & 6 & 8.2 & 0.07 & $1.37 \mathrm{~ns}$ \\
\hline HBCA & 6 & 8.7 & 0.10 & 9.0 & 6 & 9.2 & 0.07 & $4.52^{* * *}$ \\
\hline$M^{3}-M^{3}$ & 6 & 10.1 & 0.13 & 11.0 & 6 & 10.7 & 0.07 & $3.97 * *$ \\
\hline$C-M^{3}$ & 6 & 9.5 & 0.11 & 10.4 & 6 & 10.3 & 0.12 & $4.92^{* * *}$ \\
\hline$C^{1}-C^{1}$ & 6 & 6.2 & 0.14 & 6.5 & 6 & 6.6 & 0.10 & $2.23^{*}$ \\
\hline POCO & 6 & 4.8 & 0.08 & 4.7 & 6 & 4.7 & 0.06 & $0.50 \mathrm{~ns}$ \\
\hline $\mathrm{BBC1}$ & 5 & 10.8 & 0.14 & 11.1 & 5 & 11.1 & 0.09 & $2.30 *$ \\
\hline BBC2 & 6 & 11.5 & 0.09 & 12.3 & 6 & 12.2 & 0.13 & $4.60^{* * *}$ \\
\hline
\end{tabular}

BRITISH HONDURAS. Rockstone Pond ( $17^{\circ} 46^{\prime} \mathrm{N}, 88^{\circ} 22^{\prime} \mathrm{W}$ ), 1 ( ROM).

COLOMBIA. Antioquia: Medellín $\left(6^{\circ} 15^{\prime} \mathrm{N}\right.$, $\left.75^{\circ} 36^{\prime} \mathrm{W}\right), 1$ (BMNH).

Bolívar: Mompós $\left(9^{\circ} 15^{\prime} \mathrm{N}, 74^{\circ} 35^{\prime} \mathrm{W}\right)$, 2 (USNM).

Cauca: Popayán $\left(2^{\circ} 27^{\prime} \mathrm{N}, 76^{\circ} 22^{\prime} \mathrm{W}\right), 1$ (ROM).

Magdalena: Don Diego $\left(11^{\circ} 00^{\prime} \mathrm{N}, 73^{\circ}\right.$ 43'W), $10(\mathrm{~cm})$.

Meta: Guiacaramo $\left(4^{\circ} 35^{\prime} \mathrm{N}, 73^{\circ} 00^{\prime} \mathrm{W}\right)$, 4 ( $\mathrm{MCZ}), 1$ ( $\mathrm{AMNH})$; Villavicencio $\left(4^{\circ} 09^{\prime} \mathrm{N}\right.$, $\left.73^{\circ} 38^{\prime} \mathrm{W}\right), 1$ (ROM), 2 (FMNH), 4 ( AMNH).
Nariño: Barbacoas $\left(1^{\circ} 38^{\prime} \mathrm{N}, 78^{\circ} 08^{\prime} \mathrm{W}\right)$, 8 (AMNH).

Valle del Cauca: Puerto Merizalde $\left(3^{\circ} 16^{\prime}\right.$ $\mathrm{N}, 77^{\circ} 25^{\prime} \mathrm{W}$ ), 1 (USNM).

COSTA RICA. Alajuela: $18 \mathrm{~km}$ NE Naranjo $\left(9^{\circ} 56^{\prime} \mathrm{N}, 84^{\circ} 57^{\prime} \mathrm{W}\right), 15$ (TCWC).

Cartago: Turrialba $\left(9^{\circ} 56^{\prime} \mathrm{N}, 83^{\circ} 40^{\prime} \mathrm{W}\right)$, 11 ( $\mathrm{LACM}$ ), 2 ( $\mathrm{KU}), 32$ ( TCWC).

Puntarenas: Villa Neily $\left(10^{\circ} 00^{\prime} \mathrm{N}, 84^{\circ}\right.$ $\left.50^{\prime} \mathrm{W}\right), 2$ ( LACM); San José $\left(9^{\circ} 59^{\prime} \mathrm{N}, 84^{\circ}\right.$ 04'W), 1 (KU).

ECUADOR. Guayas: Balzar $\left(1^{\circ} 25^{\prime} \mathrm{S}, 79^{\circ}\right.$ $\left.54^{\prime} \mathrm{W}\right), 1$ (MNHN). 


\begin{tabular}{|c|c|c|c|c|c|c|c|}
\hline \multirow[b]{2}{*}{ Character } & \multicolumn{3}{|c|}{ E. a. auripendulus } & \multicolumn{3}{|c|}{ E. a. major } & \multirow{2}{*}{$\begin{array}{c}\text { Student's } \\
t \text { value }\end{array}$} \\
\hline & $\mathrm{n}$ & Mean & \pm S.E. & $\mathrm{n}$ & Mean & \pm S.E. & \\
\hline FOAR & 43 & 58.1 & 0.21 & 10 & 63.4 & 0.47 & $10.80 * * *$ \\
\hline 3DME & 47 & 60.2 & 0.24 & 10 & 65.6 & 0.60 & $9.07 * * *$ \\
\hline 1PHL & 46 & 25.4 & 0.16 & 10 & 27.8 & 0.43 & $5.89^{* * *}$ \\
\hline 2PHL & 47 & 23.2 & 0.19 & 10 & 25.1 & 0.18 & $7.26^{* * *}$ \\
\hline 4DME & 47 & 57.9 & 0.25 & 10 & 63.1 & 0.56 & $8.77^{* * *}$ \\
\hline 1PHL & 46 & 21.4 & 0.15 & 10 & 23.6 & 0.32 & $6.14^{* * *}$ \\
\hline 2PHL & 47 & 5.5 & 0.08 & 10 & 6.4 & 0.23 & $3.81 * * *$ \\
\hline 5DME & 47 & 32.6 & 0.16 & 10 & 35.8 & 0.17 & $13.75 * * *$ \\
\hline $1 \mathrm{PHL}$ & 47 & 17.8 & 0.13 & 10 & 19.1 & 0.13 & $7.40 * * *$ \\
\hline 2PHL & 47 & 5.6 & 0.08 & 10 & 5.7 & 0.11 & $1.03 \mathrm{~ns}$ \\
\hline TLEN & 6 & 132.8 & 2.45 & 6 & 142.5 & 1.71 & $3.23^{* *}$ \\
\hline TVER & 17 & 45.2 & 0.79 & 7 & 53.0 & 2.64 & $2.85^{*}$ \\
\hline HDFT & 28 & 11.0 & 0.32 & 9 & 11.9 & 0.65 & $1.25 \mathrm{~ns}$ \\
\hline EARS & 21 & 20.2 & 0.20 & 8 & 21.2 & 0.63 & $1.45 \mathrm{~ns}$ \\
\hline TRAG & 3 & 1.8 & 0.17 & 6 & 3.2 & 0.54 & $2.35 \mathrm{~ns}$ \\
\hline TIBI & 41 & 16.0 & 0.12 & 10 & 18.1 & 0.30 & $7.33^{* * *}$ \\
\hline HTLC & 12 & 4.0 & 0.05 & 7 & 4.6 & 0.08 & $5.90^{* * *}$ \\
\hline HTUC & 13 & 4.1 & 0.07 & 7 & 4.5 & 0.06 & $4.27^{* * *}$ \\
\hline WBSP & 11 & 0.6 & 0.06 & 9 & 0.8 & 0.05 & $2.56^{*}$ \\
\hline LBSP & 11 & 1.8 & 0.09 & 9 & 2.0 & 0.07 & $1.71 \mathrm{~ns}$ \\
\hline CIMA & 10 & 16.8 & 0.12 & 9 & 18.2 & 0.14 & $7.94^{* * *}$ \\
\hline GRLG & 13 & 17.3 & 0.09 & 10 & 18.7 & 0.09 & $10.94^{* * *}$ \\
\hline $\mathrm{C}-\mathrm{M}_{3}$ & 13 & 10.4 & 0.07 & 10 & 11.2 & 0.09 & $7.69^{* * *}$ \\
\hline$C_{1}-C_{1}$ & 12 & 3.7 & 0.05 & 9 & 3.9 & 0.04 & $3.97^{* * *}$ \\
\hline TOLG & 11 & 24.6 & 0.14 & 8 & 26.6 & 0.29 & $6.29^{* * *}$ \\
\hline CBLG & 10 & 22.8 & 0.14 & 8 & 24.8 & 0.19 & $8.45^{* * *}$ \\
\hline PALA & 12 & 10.9 & 0.07 & 10 & 11.7 & 0.15 & $5.22^{* * *}$ \\
\hline ZYGO & 9 & 14.4 & 0.08 & 8 & 15.1 & 0.11 & $5.12^{* * *}$ \\
\hline MAST & 9 & 12.3 & 0.05 & 8 & 13.0 & 0.12 & $5.23^{* *}$ \\
\hline LACH & 12 & 7.9 & 0.08 & 10 & 8.4 & 0.08 & $3.72^{* *}$ \\
\hline IOWI & 12 & 7.6 & 0.08 & 10 & 8.0 & 0.08 & $3.78^{* *}$ \\
\hline HBCA & 10 & 8.4 & 0.06 & 8 & 8.9 & 0.10 & $4.42^{* * *}$ \\
\hline$M^{3}-M^{3}$ & 12 & 9.9 & 0.05 & 10 & 10.5 & 0.08 & $5.95^{* * *}$ \\
\hline $\mathrm{C}-\mathrm{M}^{3}$ & 13 & 9.4 & 0.05 & 9 & 10.1 & 0.08 & $8.43^{* * *}$ \\
\hline$C^{1}-C^{1}$ & 11 & 6.0 & 0.06 & 10 & 6.4 & 0.05 & $5.38^{* * *}$ \\
\hline POCO & 12 & 4.6 & 0.05 & 9 & 4.9 & 0.07 & $3.20^{* *}$ \\
\hline $\mathrm{BBC} 1$ & 9 & 10.7 & 0.10 & 6 & 10.9 & 0.07 & $1.59 \mathrm{~ns}$ \\
\hline BBC2 & 10 & 11.6 & 0.07 & 8 & 12.1 & 0.18 & $2.89^{*}$ \\
\hline
\end{tabular}

Manabi: Rio Mongoya $\left(0^{\circ} 10^{\prime} \mathrm{S}, 79^{\circ} 38^{\prime}\right.$ W), 3 ( FMNH).

EL SALVADOR. Sonsonate: Chilata $\left(13^{\circ}\right.$ $40^{\prime} \mathrm{N}, 89^{\circ} 32^{\prime} \mathrm{W}$ ), 1 ( $\mathrm{MVZ}$ ).

FRENCH GUIANA. Cayenne $\left(4^{\circ} 55^{\prime} \mathrm{N}\right.$, $52^{\circ} 18^{\prime} \mathrm{W}$ ), 2 ( MNHN).

GUATEMALA. Cobán $\left(15^{\circ} 28^{\prime} \mathrm{N}, 90^{\circ} 20^{\prime}\right.$ W), 1 ( вMNH) ; Mazatenango (Finca Ciprés) $\left(14^{\circ} 32^{\prime} \mathrm{N}, 91^{\circ} 30^{\prime} \mathrm{W}\right), 1$ ( AMNH).

GUYANA. Demerara: Demerara (Georgetown) $\left(6^{\circ} 46^{\prime} \mathrm{N}, 58^{\circ} 10^{\prime} \mathrm{W}\right), 1$ (BMNH).

Essequibo: Rupununi, Kanashen ( $1^{\circ} 45^{\prime}$

\section{$\left.\mathrm{N}, 58^{\circ} 30^{\prime} \mathrm{W}\right), 1$ ( RоM).}

North West District: Arakaka $\left(7^{\circ} 35^{\prime} \mathrm{N}\right.$, $\left.60^{\circ} 07^{\prime} \mathrm{W}\right), 26$ (AMNH); Mt. Everard, 3 (AMNH).

HONDURAS. Copán: Copán (Ruinas de Copán) $\left(14^{\circ} 52^{\prime} \mathrm{N}, 89^{\circ} 10^{\prime} \mathrm{W}\right), 9$ (TCWC).

$7 \mathrm{~km} \mathrm{~N}$ Santa Bárbara $\left(14^{\circ} 56^{\prime} \mathrm{N}, 88^{\circ} 11^{\prime}\right.$ W), 1 ( TCWC).

JAMAICA. no locality, 1 (BMNH). Kingston $\left(17^{\circ} 58^{\prime} \mathrm{N}, 76^{\circ} 48^{\prime} \mathrm{W}\right), 1$ (IJ).

MEXICO. Oaxaca: Mazatlán, District of Mixes $\left(17^{\circ} 02^{\prime} \mathrm{N}, 95^{\circ} 25^{\prime} \mathrm{W}\right), 1$ (AMNH). 
Quintana Roo: 16 km from Yucatán border, 1 (UNAM).

Tabasco: Teapa $\left(18^{\circ} 35^{\prime} \mathrm{N}, 92^{\circ} 56^{\prime} \mathrm{W}\right), 1$ (BMNH).

NICARAGUA. Mecatepe $\left(11^{\circ} 58^{\prime} \mathrm{N}, 85^{\circ}\right.$ $\left.59^{\prime} \mathrm{W}\right), 1$ (USNM). $1.5 \mathrm{~km}$ SE Yalaguina $\left(13^{\circ} 20^{\prime} \mathrm{N}, 86^{\circ} 30^{\prime} \mathrm{W}\right), 1$ ( TCWC).

PANAMA. Bocas del Toro (archipelago): $7 \mathrm{~km}$ SSW Changuinola $\left(9^{\circ} 28^{\prime} \mathrm{N}, 82^{\circ} 31^{\prime} \mathrm{W}\right)$,

1 (USNM).

Canal Zone: Bohío $\left(9^{\circ} 10^{\prime} \mathrm{N}, 79^{\circ} 51^{\prime} \mathrm{W}\right)$, 13 (USNM); Empire $\left(9^{\circ} 04^{\prime} \mathrm{N}, 79^{\circ} 40^{\prime} \mathrm{W}\right)$, 1 (USNM); Fort Clayton (Fort Kobbe) $\left(9^{\circ} 00^{\prime} \mathrm{N}, 79^{\circ} 35^{\prime} \mathrm{W}\right), 2$ (USNM); Frijoles $\left(9^{\circ} 11^{\prime} \mathrm{N}, 79^{\circ} 48^{\prime} \mathrm{W}\right), 1$ (USNM); Gamboa $\left(9^{\circ} 08^{\prime} \mathrm{N}, 79^{\circ} 42^{\prime} \mathrm{W}\right), 1$ ( $\left.\mathrm{MVZ}\right)$; Panama City $\left(8^{\circ} 57^{\prime} \mathrm{N}, 79^{\circ} 30^{\prime} \mathrm{W}\right), 1(\mathrm{MCZ})$; Paraiso $\left(9^{\circ}\right.$ $02^{\prime} \mathrm{N}, 79^{\circ} 38^{\prime} \mathrm{W}$ ), 1 (USNM); Summit $\left(9^{\circ}\right.$ $04^{\prime} \mathrm{N}, 7^{\circ} 39^{\prime} \mathrm{W}$ ), 1 (USNM).

Darién: Tacarcuna Village, 1 (USNM).

Panamá: Tapia $\left(9^{\circ} 04^{\prime} \mathrm{N}, 79^{\circ} 25^{\prime} \mathrm{W}\right), 5$ (MCZ).

PERU. Huánuco: Hacienda San Antonio, Río Chinchao $\left(9^{\circ} 30^{\prime} \mathrm{S}, 75^{\circ} 56^{\prime} \mathrm{W}\right), 2$ (FMNH); $3.2 \mathrm{~km} \mathrm{~N}$ Tingo María $\left(9^{\circ} 09^{\prime} \mathrm{S}\right.$, $\left.75^{\circ} 56^{\prime} \mathrm{W}\right), 1$ ( TCWC) ; Vista Alegre $\left(9^{\circ} 55^{\prime} \mathrm{S}\right.$, $\left.76^{\circ} 11^{\prime} \mathrm{W}\right), 2$ (FMNH).

Junín: Perené $\left(10^{\circ} 55^{\prime} \mathrm{S}, 75^{\circ} 15^{\prime} \mathrm{W}\right), 5$ (USNM).

Loreto: Pucallpa $\left(8^{\circ} 23^{\prime} \mathrm{S}, 74^{\circ} 32^{\prime} \mathrm{W}\right), 1$ (FMNH).

Piura: $6.4 \mathrm{~km} \mathrm{~W}$ Suyo $\left(4^{\circ} 33^{\prime} \mathrm{S}, 80^{\circ} 01^{\prime}\right.$ W), 2 ( TCWC).

San Martín: Guayabamba (Santa Rosa de Huayabamba $) \quad\left(6^{\circ} 22^{\prime} \mathrm{S}, \quad 77^{\circ} 25^{\prime} \mathrm{W}\right), \quad 4$ (AMNH).

SURINAM. Commewijne: Slootwijk (approx. $\left.5^{\circ} \mathrm{N}, 55^{\circ} \mathrm{W}\right), 1$ (RMNH).

Nickerie: Alalapadoo, $\mathrm{N}$ of Sipaliwini R. (approx. $2^{\circ} 30^{\prime} \mathrm{N}, 56^{\circ} \mathrm{W}$ ) , 6 (ROM). Plantation Wederzorg, not located, 1 (USNM).

Surinam: no locality, 1 (USNM); Paramaribo $\left(5^{\circ} 52^{\prime} \mathrm{N}, 55^{\circ} 14^{\prime} \mathrm{W}\right), 2(\mathrm{RMNH})$. Welgedacht C, S of Paramaribo, 1 (RMNH).

TRINIDAD. Blanchisseuse $\left(10^{\circ} 47^{\prime} \mathrm{N}, 61^{\circ}\right.$ $18^{\prime} \mathrm{W}$ ), 1 (USNM).

VENEZUELA. Amazonas: San Fernando de Atabapo, Upper Orinoco River $\left(4^{\circ} 03^{\prime} \mathrm{N}\right.$, $\left.67^{\circ} 42^{\prime} \mathrm{W}\right), 2$ (AMNH).

Bolívar: El Dorado, Río Cuyuni $\left(6^{\circ} 45^{\prime} \mathrm{N}\right.$, $\left.61^{\circ} 37^{\prime} \mathrm{W}\right), 9$ (AMNH).

Carabobo: $10 \mathrm{~km}$ NW Urama, Río Yaracuy $\left(10^{\circ} 30^{\prime} \mathrm{N}, 68^{\circ} 19^{\prime} \mathrm{W}\right), 4$ (USNM).

Guárico: Parapara $\left(9^{\circ} 44^{\prime} \mathrm{N}, 67^{\circ} 18^{\prime} \mathrm{W}\right)$, 1 (USNM).

Zulia: Lagunillas $\left(10^{\circ} 08^{\prime} \mathrm{N}, 71^{\circ} 16^{\prime} \mathrm{W}\right)$, 1 (FMNH).

Acknowledgments - I thank the following curators in charge of mammal collections for loan of specimens in their care: Dr. Karl F. Koopman, AMnH; Dr. Frank B. Gill, ANSP; Mr. Kenneth Doutt, CM; Dr. Robert S. Hoffman, KU; Mr. Thomas H. Farr, I J Dr. Donald R. Patten, LACM; Dr. Barbara Lawrence, MCZ; Dr. Andre Brosset, MNHN, Brunoy; Dr. Jean Dorst, Mnhn, Paris; Dr. James L. Patton, MVZ; Dr. A. M. Husson, RMnH; Dr. David J. Schimdly, TCwC; Dr. Helio Ferraz de Almeida Camargo, UsP; and Dr. José Ramírez Pulido, UNAM. I am also grateful to Mr. John E. Hill, BMnн; Dr. Philip Hershkovitz, FMNH; and Dr. Charles O. Handley, USNM for the opportunity to examine specimens in their charge. I also thank Dr. B. V. Peterson, Entomology Research Institute, Ottawa, who was instrumental in arranging the loan of specimens from Universidade de São Paulo.

Drs. J.R. Tamsitt and D. Valdivieso read the manuscript critically, and Dr. R.L. Peterson gave valuable advice and counsel. I thank Mr. Jim Borack for photographic services and the staff of the Department of Mammalogy for their assistance. This study was supported in part by a National Research Council of Canada grant to Dr. R. L. Peterson and by the Royal Ontario Museum. I acknowledge the use of the photographic facilities of the Laboratory of Analytical Systematics in the Royal Ontario Museum established through a grant from the National Research Council of Canada to the Department of Zoology, University of Toronto.

Resumen-El murciélago mastín, Eumops auripendulus (Chiroptera: Molossidae) tiene una distribución desde México hasta Argentina. Cierta población del este del Brasil y del noreste de Argentina difiere morfológicamente de E. a. auripendulus. Describo aquí 
tales quirópteros como una nueva subespecie, E. a. major, la cúal se encuentra geográficamente aislada de la subespecie de menor tamaño $E$. a. auripendulus, que se distribuye desde la cuenca amazónica hasta el sur de México. La localidad típica de E. a. major es Campo Viera $\left(27^{\circ} 15^{\prime} \mathrm{S}, 55^{\circ} 10^{\prime} \mathrm{W}\right)$ en Misiones, Argentina. El color del pelaje de esta nueva subespecie es similar al de $E$. $a$. auripendulus pero $E$. a. major es significantemente de mayor tamaño $(P \leqslant 0.05)$ en 27 de las 38 características mensurales en los machos y en 31 de 38 en las hembras. Las características que distinguen principalmente estas dos subespecies son: la longitud del antebrazo y del tercer metacarpo; la longitud condiloincisiva y de la mandíbula; la longitud de la hilera inferior de dientes; la longitud mayor y la condiloincisiva del cráneo y la longitud superior de dientes, pero esta última característica de diferenciación se aplica solamente a las hembras.

\section{Literature Cited}

BUFFON, G.L.L.

1789 Histoire naturelle générale et particulière. Suppl., tome 7. Paris, De l'Imprimerie royale. $364 \mathrm{pp}$.

EGER, J.L.

1973 Systematics of the genus Eumops (Chiroptera: Molossidae). M.Sc. thesis, University of Toronto. $158 \mathrm{pp}$.

GOODWIN, G.G.

1960 The status of Vespertilio auripendulus Shaw, 1800, and Molossus ater Geoffroy, 1805. Am. Mus. Nov., 1994: 1-6.

HUSSON, A.M.

1962 The bats of Suriname. Zool.Verh., Leiden, 58: 1-282.

PENNANT, T.

1793 History of quadrupeds. 3rd ed. Vol. 2. London, B. \& J. White. 324 pp. RIDGWAY, R.

1912 Color standards and color nomenclature. Washington, D.C., Published by the author. 43 pp.

SANBORN, C.C.

1932 Bats of the Genus Eumops. J. Mammal. 13 (4) : 347-357.

SHAW, G.

1800 General zoology; or systematic natural history. Vol. 1, pt. 1. Mammalia. London, G. Kearsley. 248 pp.

SOKAL, R.R. AND F.J. ROHLF

1969 Biometry. San Francisco, W.H. Freeman. 776 pp.

TEMMINCK, C.J.

1827 Monographies de mammalogie, ou Description de quelques genres de mammifères, dont les espéces ont été observées dans les differens musées de l'Europe. Vol. 1, pt. 1. Paris, G. Dufour et d'Ocogne.

THOMAS, O.

1920 A further collection of mammals from Jujuy. Ann. Mag. Nat. Hist. 9(5): 188-196. Suggested citation: Life Sci. Occ. Pap., R. Ont. Mus.

All manuscripts considered for publication are subject to the scrutiny and editorial policies of the Life Sciences Editorial Board and to review by persons other than Museum staff who are authorities in the particular field involved.

PRICE: 50 c

(C) The Royal Ontario Museum, 1974

100 Queen's Park, Toronto, Canada M5S 2C6

PRINTED AT THE UNIVERSITY OF TORONTO PRESS

ISBN: 0-88854-161-9 




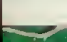




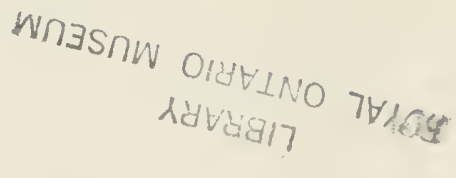

. TÁL ONTARIO MUSEUA 

a. 